\title{
Особенности сделок слияний
}

\section{и поглощений инновационных компаний на развитых}

\section{и развивающихся рынках капитала}

\author{
Ирина Вячеславовна Скворцова \\ Преподаватель, Факультет экономических наук, Школа финансов \\ Национальный исследовательский университет «Высшая школа экономики» \\ Москва, ул. Мясницкая, д. 20 \\ E-mail: irina.v.skvortsova@gmail.com
}

\author{
Андрей Дмитриевич Красовицкий \\ Стажер-исследователь, научно-учебная лаборатория корпоративных финансов \\ Национальный исследовательский университет «Высшая школа экономики» \\ Москва, ул. Мясницкая, д. 20 \\ E-mail: andreykras99@gmail.com
}

\begin{abstract}
Аннотация
В настоящее время инновационные компании являются одним из ключевых драйверов мирового рынка слияний и поглощений, что обусловливает актуальность выявления их особенностей, а также исследования вопросов эффективности подобных сделок. Вместе с тем в современной академической литературе отсутствуют однозначные ответы на вопросы о том, какую компанию можно считать инновационной, каким образом определить уровень ее инновационности, что такое инновационная сделка по слиянию и поглощению и какими особенностями она обладает. Крайне мало внимания уделяется международным инновационным сделкам по слияниям и поглощениям и определению их особенностей. В данной статье приводится краткий обзор имеющейся литературы на данную тему. Описываются способы определения инновационной компании и основные подходы для оценки эффективности инновационных сделок по слияниям и поглощениям. Рассматриваются также международные инновационные сделки по слияниям и поглощениям и их особенности. По результатам проведенного обзора был получен вывод о том, что в большинстве работ инновационность определяется через абсолютную величину различных показателей, список которых приводится в данной статье, или с помощью специальных опросников, которые направляются менеджменту компании. Инновационные компании также могут быть определены исходя из отраслей, в которых задействованы компании. Для определения эффективности инновационных сделок по слияниям и поглощениям, как правило, используются показатели, связанные с приростом или цитируемостью имеющихся патентов у компании. Однозначного ответа на вопрос о влиянии сделок по слияниям и поглощениям на инновационную активность компании дать не удалось, так как результаты исследований имеют противоречивый характер. Основным же мотивом международных инновационных сделок по слияниям и поглощениям является приобретение новых технологий для получения конкурентных преимуществ. Тем не менее полное представление об эффективности данного типа сделок отсутствует.
\end{abstract}

Ключевые слова: слияния и поглощения, инновационные компании, инновации, инновационная эффективность, эффективность деятельности компании, развитые рынки, развивающиеся рынки

JEL: G34, L10, O30, O31 


\section{Подходы к определению инновационной компании и уровня инновационности}

В общем виде под инновациями понимается внедрение новых идей или оригинальных решений, т.е. введение значительных технических, технологических, коммерческих, маркетинговых, финансовых, социальных (например, в человеческих ресурсах), а также организационных изменений продукта или процесса [1-6]). Соответственно инновационными компаниями считаются те, которые занимаются разработкой инноваций или активно задействуют их в своих внутренних бизнес-процессах.

В научной литературе отсутствует единая точка зрения относительно того, что представляют собой инновации. Так, в работе А. Субраманина и С. Нилаканта [7] под инновациями понимается реакция компании на изменения во внешней среде ее функционирования. Дж. Азар и Ф. Чиабуши [8] делят все инновации на технологические и организационные. Под технологической инновацией понимается реализация идеи для нового продукта и услуги или внедрение новых элементов в процессы производства или обслуживания. Организационная же инновация определяется как создание и внедрение практики, процесса, структуры или техники управления, которые являются новыми для организационной структуры компании и предназначены для достижения дальнейших организационных целей. Инновации могут также рассматриваться как процесс внесения изменений в уже существующий продукт или процесс, что повышает их ценность для клиентов [9].

По определению Организации экономического сотрудничества и развития (ОЭСР) инновационной следует считать фирму, которая осуществляла инновационную деятельность в течение рассматриваемого периода вне зависимости от того, привела ли данная деятельность к внедрению инноваций [11]. Под инновационной деятельностью подразумеваются все научные, технологические, организационные, финансовые и коммерческие инициативы и направления деятельности в компании, целью которых является внедрение инноваций. Данная деятельность также включает в себя исследования и разработки, которые напрямую не связаны с конкретными инновациями.

Некоторые авторы не различают инновационные и высокотехнологичные компании (см. например, [10]). В соответствии с классификацией ОЭСР, высокотехнологичными считаются компании с высокой долей расходов на НИОКР от выручки ОЭСР. Иначе говоря, это те компании, деятельность которых в первую очередь связана с разработкой инноваций.
В академической литературе приводят множество различных способов определения степени инновационности фирмы. В большинстве работ инновационность определяется через абсолютную величину различных показателей: чем взятый показатель больше, тем выше инновационность компании $[10 ; 12 ; 13])$. Например, чем выше уровень расходов на НИОКР или чем больше патентов получает компания, тем выше уровень ее инновационности. К таким показателям можно также отнести:

- расходы компании на НИОКР;

- $\quad$ количество патентов;

- количество раз, когда патенты фирмы использовались при создании других патентов;

- разнообразие продаваемых товаров.

Существенным недостатком вышеуказанных показателей является то, что они не учитывают особенности компаний (например, размер). В связи с этим зачастую используются специальные индексы или нормированные показатели ([14-16]; BCG, 2018¹). К их числу необходимо отнести следующие показатели:

- количество «цитирований» патентов (сколько раз патенты использовались при создании других патентов) для каждой компании, деленное на среднее значение «цитирований» для индустрии;

- специальные опросники, направляемые руководителям научно-исследовательских подразделений компаний, которые отвечают на вопросы, связанные с инновационностью (примеры вопросов: «Ваша компания является инновационной?», «Насколько высока вероятность создания нового продукта?», «У Вас в компании часто происходят инновационные нововведения?»)2;

- отношение расходов к общей выручке компании;

- интеллектуальный капитал, который измеряется по следующей формуле:

$I C_{t}=\alpha\left(H_{t}+S_{t}+R_{t}\right)+(1-\delta) I C_{t-1}$,

где $I C_{t}$ - интеллектуальный капитал; $H_{t}$ - человеческий капитал; $S_{t}$ - структурный капитал; $R_{t}$ - отношенческий капитал; $\alpha$ - коэффициент накопления; $\delta$ - коэффициент амортизации.

Важно обратить внимание на то, что большинство вышеприведенных показателей связаны с технологическими инновациями или инновациями в бизнес-процессах (например, новый способ производства). В случае если инновации касаются новых подходов к ведению маркетинговой деятельности (дизайн продукта, его продвижение и т.д.) или организационной структуры компании, возможности

\footnotetext{
${ }^{1}$ URL: https://www.bcg.com/ru-ru/publications/collections/most-innovative-companies-2018.aspx

${ }^{2}$ В большинстве случаев ответы на вопросы имеют семибалльную шкалу (от «строго не согласен» до «строго согласен»). Далее на основе полученных ответов выводятся индексы (в найденной литературе методология не уточняется).
} 
проведения масштабных эмпирических исследований существенно снижаются, поскольку для них в большинстве случаев необходимы специальные опросники, которые следует направлять в компании.

В табл. 1 представлена классификация показателей, которые могут быть использованы для оценки степени инновационности фирмы в зависимости от того, насколько данные показатели могут быть применены при проведении эмпирического исследования.

Таблица 1. Подходы к оценке уровня инновационности фирмы

Возможность измерения и использования

в эмпирическом исследовании - высокая

\begin{tabular}{|c|c|}
\hline Показатель & Автор(ы) \\
\hline Расходы на НИОКР & \\
\hline $\begin{array}{l}\text { Доля расходов на НИОКР в } \\
\text { выручке }\end{array}$ & {$[10 ; 12 ; 13 ; 17-19]$} \\
\hline Количество патентов & {$[10 ; 20 ; 13]$} \\
\hline
\end{tabular}

Возможность измерения и использования в эмпирическом исследовании - средняя

Сколько раз патенты фирмы использовались для создания других патентов (количество $[10 ; 14 ; 20]$ «цитирований патента»)

Интеллектуальный капитал: человеческий капитал, структурный капитал и отношенче-

ский капитал

\begin{tabular}{|c|c|}
\hline $\begin{array}{l}\text { Разнообразие продаваемых } \\
\text { продуктов }\end{array}$ & {$[10 ; 13 ; 22]$} \\
\hline $\begin{array}{l}\text { Создание нового продукта, } \\
\text { бизнес-процесса }\end{array}$ & {$[19 ; 23-26]$} \\
\hline
\end{tabular}

\section{Возможность измерения и использования в эмпирическом исследовании - низкая}

Нематериальные активы: знания и опыт, организационные системы и процессы, инно$[21 ; 27 ; 28]$ вации и технологии, деловые связи

\begin{tabular}{l}
$\begin{array}{l}\text { Возможность создания ново- } \\
\text { го продукта, идеи }\end{array}$ \\
\hdashline $\begin{array}{l}\text { Доля выручки от реализации } \\
\text { нового продукта }\end{array}$ \\
\hdashline $\begin{array}{l}\text { Специальные опросники, } \\
\text { которые направляются менед- } \\
\text { жерам компаний }\end{array}$
\end{tabular}

\section{Мотивы сделок по слияниям}

\section{и поглощениям инновационных компаний}

С одной стороны, мотивы инновационных сделок по слияниям и поглощениям совпадают с мотивами обычных сделок. Например, компания может быть заинтересована в улучшении своей организационной структуры [31], что положительно влияет на показатели компании. Сделки инновационных компаний позволяют сократить издержки благодаря эффекту масштаба [32; 33], быстро выйти на новые рынки [34; 35], перераспределить ресурсы, в том числе занятые в сфере НИОКР [36], увеличить клиентскую базу и базу поставщиков [37; 38]), повысить рыночную власть [32; 39; 40], снизить величину налогов и стоимость капитала, диверсифицировать денежные потоки [39; 41; 42]) и т.д.

С другой стороны, инновационные сделки по слияниям и поглощениям имеют свои специфические мотивы. В большинстве случаев основным из них является поглощение внешних технологических возможностей с целью успешной конкуренции в современных экономических условиях [43; 44]. Иначе говоря, основной целью инновационных сделок является расширение имеющейся базы знаний компании. Такие сделки позволяют также разнообразить линейку выпускаемых товаров, в том числе за счет совмещения инновационных возможностей $[45 ; 46]$.

Принимая во внимание высокую популярность сделок слияний и поглощений среди инновационных компаний, логично предположить существенное улучшение результатов деятельности в результате подобных сделок. При этом многие эмпирические исследования, посвященные влиянию сделок слияний и поглощений на эффективность деятельности инновационных фирм, демонстрируют противоречивые результаты [33].

Кроме того, стоит обратить внимание на то, что выше были рассмотрены только те мотивы, которые оказывают положительное влияние на благосостояние акционеров компании, однако нельзя забывать, что сделки слияний и поглощений могут также оказывать и отрицательное влияние на благосостояние акционеров. В частности, в соответствии с hubris hypothesis ${ }^{3}$, самоуверенные менеджеры могут совершать сделки по завышенной цене [47]. Кроме того, менеджеры могут в ряде случаев стремиться осуществлять те сделки, эффективность которых в наибольшей степени зависит от их специфических умений и способностей [48], что может стать причиной совершения невыгодных сделок. Участие в сделках слияний и поглощений отвлекает менеджеров от их основной деятельности, что может отрицательным образом влиять на долгосрочные инвестиции в НИОКР и, следовательно, в целом на инновационную деятельность компании.

${ }^{3}$ В соответствии с данной гипотезой компания-покупатель имеет тенденцию совершать сделки по завышенной цене. 


\section{Способы оценки эффективности сделок по слияниям и поглоще- ниям инновационных компаний}

Что касается подходов к оценке влияния инновационных сделок по слияниям и поглощениям на эффективность деятельности компании, то они не отличаются от подходов, используемых для оценки влияния обычных сделок по слияниям и поглощениям. К ним также могут быть применены такие традиционные методы, как event study, accounting study, case study, которые используются при оценке эффективности сделок по слияниям и поглощениям обычных компаний. Единственной особенностью являются детерминанты сделок и используемые контрольные переменные, такие как, например, расходы на НИОКР [49].
В современных академических работах большое внимание уделяется вопросам технологической эффективности инновационных сделок слияний и поглощений. В большинстве из них применяется регрессионный анализ панельных данных [45; 50-54]. В качестве зависимой переменной принято использовать показатели инновационности компании (см. табл. 1), причем самыми популярными из них являются показатели, связанные с патентами и долей расходов на НИОКР в выручке. В исследованиях принято рассматривать динамику показателей во времени и в качестве объясняющих переменных использовать их значения с лагом. В табл. 2 приведен анализ ключевых детерминант эффективности сделок слияний и поглощений инновационных компаний, выполненный на примере крупнейших современных академических исследований в области инновационной эффективности компаний.

Таблица 2. Ключевые детерминанты эффективности сделок слияний и поглощений инновационных компаний

\begin{tabular}{|c|c|c|c|c|}
\hline Автор(ы) & Выборка & $\begin{array}{l}\text { Зависимая } \\
\text { переменная }\end{array}$ & Объясняющая переменная & $\begin{array}{c}\text { Влияние } \\
\text { на зависимую } \\
\text { переменную }\end{array}$ \\
\hline \multirow{3}{*}[45]{} & \multirow{3}{*}{$\begin{array}{l}72 \text { крупнейшие междуна- } \\
\text { родные фирмы (30 компа- } \\
\text { ний представляют Европу, } \\
26 \text { - Америку, } 16 \text { - Японию) } \\
\text { из химической индустрии } \\
\text { за период 1980-1991 гг. }\end{array}$} & \multirow{3}{*}{$\begin{array}{l}\text { Уровень иннова- } \\
\text { ционности после } \\
\text { сделки (патенты) }\end{array}$} & $\begin{array}{l}\text { Абсолютный размер погло- } \\
\text { маемой базы знаний }\end{array}$ & $(+)^{* * *}$ \\
\hline & & & $\begin{array}{l}\text { Относительный размер } \\
\text { поглощаемой базы знаний }\end{array}$ & $(+)^{* *}$ \\
\hline & & & $\begin{array}{l}\text { Нетехнологические сделки } \\
\text { слияний и поглощений }\end{array}$ & Незначим \\
\hline \multirow{3}{*}[50]{} & \multirow{3}{*}{$\begin{array}{l}201 \text { сделка за период 1986- } \\
1992 \text { гг. Выборка представ- } \\
\text { лена } 35 \text { международными } \\
\text { компаниями ( } 30 \text { из США, } \\
\text { двумя из Азии, двумя из } \\
\text { Европы) из сектора ком- } \\
\text { пьютерных технологий }\end{array}$} & \multirow{3}{*}{$\begin{array}{l}\text { Прирост патентной } \\
\text { интенсивности } \\
\text { американских па- } \\
\text { тентов компаний }\end{array}$} & $\begin{array}{l}\text { Доля связанных сделок } \\
\text { слияний и поглощений } \\
\text { от всех сделок }\end{array}$ & $(+)^{* * *}$ \\
\hline & & & $\begin{array}{l}\text { Доля технологически свя- } \\
\text { занных сделок слияний и } \\
\text { поглощений }{ }^{\text {I }} \text { от всех сделок }\end{array}$ & $(+)^{*}$ \\
\hline & & & $\begin{array}{l}\text { Интенсивность НИОКР } \\
\text { (отношение интенсивности } \\
\text { расходов на НИОКР за два } \\
\text { года до сделки к средней } \\
\text { интенсивности по отрасли) }\end{array}$ & $(+)^{* * *}$ \\
\hline & \multirow{2}{*}{$\begin{array}{l}\text { Международная выборка } \\
\text { компаний ( } 256 \text { из Северной } \\
\text { Америки, } 45 \text { из Европы, } 46 \\
\text { из Азии), охватывающая }\end{array}$} & & $\begin{array}{l}\text { Абсолютный размер погло- } \\
\text { щаемой базы знаний }\end{array}$ & $(+)^{* * *}$ \\
\hline & & & $\begin{array}{l}\text { Относительный размер } \\
\text { поглощаемой базы знаний }\end{array}$ & $(-)^{* * *}$ \\
\hline
\end{tabular}

\section{[51]}

четыре высокотехнологичные отрасли: аэрокосмическую и оборонную (21 компания), производство компьютеров и офисной техники (76 компаний), фармацевтика (77 компаний) и электронику и связь (173 компании) за период $1985-1994$ гг.
Уровень инновационности после сделки (патенты)
Нетехнологические сделки слияний и поглощений
$(-)^{* * *}$

\footnotetext{
I Слияние или поглощение компании из той же индустрии.

II Слияние или поглощение компании со схожим портфелем патентов, которые имеются в наличии у компании.
} 


$\begin{array}{lll}\text { Автор(ы) } & \text { Выборка } & \text { Вависимая } \\ & \text { переменная } & \text { Объясняющая переменная на зависимую } \\ & & \text { переменную }\end{array}$

Размещение заявок

на патенты в год

Влияние патен-

159 сделок слияний и поглощений компаний, занятых в производстве медицинского и фотографического оборудования США за период с 1988 по 1996 г. (компания-покупатель и компания-цель базируются в США)

[53]

31 горизонтальная M\&A сделка (слияние или поглощение компании из той же отрасли) европейских и американских компаний за период с 1987 по 2001 г.

та (количество

будущих цитирований, полученных после размещения патента)

Применимость патентов в других отраслях

Применимость патентов в других технологиях

Инновационная эффективность: патентная активность, создание новых технологий и скорость разработки технологических знаний (измеряется

$(+)^{*}$ по пятибалльной шкале)

Европейские производственные компании за период 1994-2002 гг.
Вероятность быть инновационным новатором в период $t$

Технологическая схожесть
$\begin{aligned} & \text { Повышение производи- } \\ & \text { тельности НИОКР после } \\ & \text { сделки слияний и поглоще- } \\ & \text { ний }\end{aligned}$

Нарушения в работе отдела

НИОКР после сделки слия- Незначим ний и поглощений
$(-)^{* *}$

$(-)^{* *}$

Вероятность перехода от новатора в момент времени $t-k$ к новатору в момент

$(+)^{* * *}$ времени $t$

Вероятность сохранения постоянной инновационной активности

$(-)^{* *}$

Сделка по M\&A

Была ли компания инновационным новатором в период $t-k$

* $10 \%$ уровень значимости зависимой переменной; ${ }^{* *} 5 \%$ уровень значимости зависимой переменной;

*** $1 \%$ уровень значимости зависимой переменной.

Анализ показателей инновационности позволяет сделать вывод о том, что наиболее часто для оценки уровня инновационности в эмпирических исследованиях используется количество патентов, которыми владеет компания. Это связано с тем, что количество патентов компании позволяет ответить на вопрос, является ли рассматриваемая компания инновационной начиная с уровня ее технологического развития и заканчивая организационной структурой компании. Следовательно, чем большим количеством патентов владеет компания, тем выше уровень ее инновационности.

\section{Эффективность сделок по слияниям и поглощениям инновационных компаний на развитых и развивающихся
рынках капитала}

Мотивы сделок слияний и поглощений, которыми руководствуются инновационные компании на развитых и развивающихся рынках капитала, в большинстве случаев различны. Если компании-покупатели 
с развитых рынков капитала чаще всего прибегают к слияниям и поглощениям в целях повышения операционной эффективности, то для покупателей, представляющих растущие рынки капитала, основной мотив заключается в быстром приобретении технологий и завоевании соответствующей рыночной доли. Результаты современных академических работ, посвященных вопросам эффективности сделок с участием инновационных компаний, свидетельствуют о противоречивых результатах как для развитых, так и для развивающихся экономик.

Для инновационных компаний-покупателей с развитых рынков капитала результатом международных сделок слияний и поглощений может стать как увеличение, так и снижение инновационной активности в зависимости от возможностей интегрирования базы знаний своей и поглощаемой компаний. В частности, М. Клудт с соавторами [51] на примере 347 международных высокотехнологичных компаний показали, что с ростом и абсолютной, и относительной величины базы знаний компании-цели инновационная активность компании-покупателя снижается. Авторы также эмпирически доказали, что в том случае, если инновационная компания из развитой страны приобретает неинновационную компанию, инновационная активность компании-покупателя, измеренная посредством количества полученных патентов, уменьшается.

На примере поглощения немецких компаний фирмами из развитых стран было выявлено, что сделки М\&А приводят к падению расходов на НИОКР компаний-целей, что, в свою очередь, отрицательно влияет на объем инноваций, в качестве прокси для которого использованы такие дамми-переменные как изобретение новых товаров и новых производственных процессов [55].

Международные сделки слияний и поглощений на европейском рынке с участием инновационных компаний приводят к увеличению общего количества патентов, полученных компанией-целью и компаниейпокупателем, однако данный эффект достигается за счет перераспределения инновационных ресурсов в пользу покупателя, что выражается в одновременном росте и снижении количества патентов покупателя и поглощенной компании [55]. Более того, эффективность сделок отрицательно зависит от уровня технологической дистанции между компанией-целью и компанией-покупателем [56].

На развитых рынках капитала сделки слияний и поглощений положительно влияют на количество получаемых патентов, но отрицательно на применимость патента в других отраслях и технологиях [52]. Результативность НИОКР после сделки слияний и поглощений оказывает положительное влияние на инновационную эффективность, тогда как влияние технологической схожести оказывается отрицательным [53].

Инновационные компании с развивающихся рынков капитала в основном приобретают готовые технологии по производству уже выпускаемых товаров, а не технологии, которые только лишь потенциально могут быть использованы при разработке инновационных продуктов [57]. Следовательно, для компаний с развивающихся рынков капитала инвестиции в развитые рынки с помощью сделок по слияниям и поглощениям связаны в первую очередь с возможностью повышения экономической эффективности, в частности, улучшения параметров используемого оборудования, сокращения издержек производства, повышения добавленной стоимости товаров и услуг. К такому выводу приходят, например, Дж. Ю и др. [58], которые на примере 114 китайских компаний показывают, что инновационность является показателем организационного потенциала для обеспечения и повышения устойчивости конкурентных преимуществ в динамичной среде развивающихся стран Азии.

Инновационные компании с растущих рынков капитала при выборе цели в сделках слияний и поглощений обращают внимание не на степень инновационности сделки, а на другие факторы, которые будут оказывать положительное воздействие на эффективность дальнейшей деятельности, повышение которой и становится одним из самых важных детерминант в сделках слияний и поглощений. В частности, Ф. Де Буеле и А. Селс [59] на примере сделок по слияниям и поглощениям высокотехнологичных европейских компаний индийскими ${ }^{4}$ (в конечную выборку вошло 147 сделок) доказали, что эффективность сделок не зависит от степени инновационности покупателя, измеренной с помощью доли расходов на НИОКР в совокупной выручке компании.

Следует также обратить внимание на то, что для сделок, в которых компания с растущего рынка капитала приобретает высокотехнологичную фирму, работающую на развитом рынке, патентная активность компании-цели не изменяется, тогда как патентная активность покупателя с развивающегося рынка растет. К такому выводу пришли, например, Дж. Андерсон и Д. Сутерланд [60] на примере китайских компаний (рассматривался период 1998-2012 гг., в выборку вошло 241 наблюдение).

Наряду с выходом на новые рынки одним из основных мотивов сделок слияний и поглощений для компаний с развивающихся рынков капитала является возможность приобретения передовых технологических и человеческих ресурсов, недоступных на локальных рынках [61] для их последующего использования на внутренних домашних рынках ([62], [57]). Так, С. Ченг и М. Янг [63] на примере 186 китайских

\footnotetext{
${ }^{4}$ На примере публичных индийских компаний, которые торгуются на европейских биржах за период с 1996 по 2010 г.
} 
компаний показали, что фирмы-покупатели с развивающихся рынков получают выгоды от своих деловых партнеров в развитых странах (в Северной Америке, Японии и странах Западной Европы), занятых в машиностроительной, электронной промышленности, иных обрабатывающих отраслях и в сфере услуг. Иначе говоря, после завершения международных сделок по слияниям и поглощениям фирмы-покупатели могут постоянно получать ресурсы и поддержку от существующих и новых партнеров фирм-целей. Чем выше уровень деловых связей, тем выше эффективность сделки по слиянию и поглощению. Авторам также удалось доказать, что чем выше уровень культурной дистанции в международных сделках слияний и поглощений, тем более отрицательное воздействие уровень инновационности оказывает на эффективность сделок.

\section{Заключение}

Подводя итог вышесказанному, можно сформулировать следующие выводы. Мотивы сделок слияний и поглощений инновационных компаний имеют свои особенности, ключевой из которых является быстрое приобретение технологий с целью успешной конкуренции. Мотивы инновационных компаний, принимающих решение о сделках слияний и поглощений, различаются в зависимости от того, работает ли фирма на развитом или развивающемся рынке капитала. Результаты современных академических исследований по данной тематике свидетельствуют о противоречивых результатах, что не позволяет сформулировать однозначного вывода об эффективности подобных сделок.

\section{Список литературы}

1. Quinn J.B. Technological innovation, entrepreneurship, and strategy. IEEE Engineering Management Review. 1983;20(3):14-25. DOI: 10.1109/EMR.1983.4306000

2. Damanpour F. Organizational innovation: A metaanalysis of effects of determinants and moderators. Academy of Management Journal. 1991;34(3):555590. DOI: $10.5465 / 256406$

3. Damanpour F. Organizational complexity and innovation: Developing and testing multiple contingency models. Management Science. 1996;42(5):693-716. DOI: 10.1287/mnsc.42.5.693

4. Tidd J., Bessant J., Pavitt K. et al. Management dell'innovazione: L'integrazione del cambiamento tecnologico, organizzativo e dei mercati. Milano: Guerini e associate; 1999. $453 \mathrm{p}$.

5. Valls J.P., Escorsa P. Tecnología e innovación en la empresa: Dirección y gestión. Barcelona: Universidad Politecnica de Cataluny; 2000. 341 p.
6. Camisón-Zornoza C., Lapiedra-Alcamí R., Segarra-Ciprés M., Boronat-Navarro M. A metaanalysis of innovation and organizational size. Organization Studies. 2004;25(3):331-361. DOI: $10.1177 / 0170840604040039$

7. Subramanian A., Nilakanta S. Organizational innovativeness: Exploring the relationship between organizational determinants of innovation, types of innovations, and measures of organizational performance. Omega. 1996;24(6):631-647. DOI: 10.1016/S0305-0483(96)00031-X

8. Azar G., Ciabuschi F. Organizational innovation, technological innovation, and export performance: The effects of innovation radicalness and extensiveness. International Business Review. 2017;26(2):324-336. DOI: 10.1016/j. ibusrev.2016.09.002

9. Leicht T., Chtourou A., Youssef K.B. Consumer innovativeness and intentioned autonomous car adoption. The Journal of High Technology Management Research. 2018;29(1):1-11. DOI: 10.1016/j.hitech.2018.04.001

10. Hagedoorn J., Cloodt M. Measuring innovative performance: is there an advantage in using multiple indicators? Research Policy. 2003;32(8):1365-1379. DOI: 10.1016/S0048-7333(02)00137-3

11. OECD, 2005. Oslo Manual, OECD, Paris.

12. Hall L.A., Bagchi-Sen S. A study of R\&D, innovation, and business performance in the Canadian biotechnology industry. Technovation. 2002;22(4):231-244. DOI: 10.1016/S01664972(01)00016-5

13. Artz K.W., Norman P.M., Hatfield D.E., Cardinal L.B. A longitudinal study of the impact of R\&D, patents, and product innovation on firm performance. The Journal of Product Innovation Management. 2010;27(5):725-740. DOI: 10.1111/j.15405885.2010.00747.x

14. Dutta S., Weiss A.M. The relationship between a firm's level of technological innovativeness and its pattern of partnership agreements. Management Science. 1997;43(3):257-402. DOI: 10.1287/ mnsc.43.3.343

15. Hult G.T.M., Hurley R.F., Knight G.A. Innovativeness: Its antecedents and impact on business performance. Industrial Marketing Management. 2004;33(5):429-438. DOI: 10.1016/j.indmarman.2003.08.015

16. Sydler R., Haefliger S., Pruksa R. Measuring intellectual capital with financial figures: Can we predict firm profitability? European Management Journal. 2014;32(2):244-259. DOI: 10.1016/j. emj.2013.01.008 
17. Fung M.K. Is innovativeness a link between pay and performance? Financial Management. 2009;38(2):411-429. DOI: 10.1111/j.1755053X.2009.01041.x

18. Leitner S.M., Stehrer R. The role of financial constraints for different innovation strategies: Evidence for CESEE and FSU countries. Wiener Institut für Internationale Wirtschaftsvergleiche. WIIW Working Paper. 2016;(125). URL: https://wiiw. ac.at/the-role-of-financial-constraints-for-differentinnovation-strategies-evidence-for-cesee-and-fsucountries-dlp-3875.pdf

19. Shi X., Wu Y. The effect of internal and external factors on innovative behaviour of Chinese manufacturing firms. China Economic Review. 2017;46(S):S50-S64. DOI: 10.1016/j. chieco.2016.08.010

20. Aghion P., Bloom N., Blundell R., Griffith R., Howitt P. Competition and innovation: An inverted-U relationship. The Quarterly Journal of Economics. 2005;120(2):701-728. DOI: 10.1093/qje/120.2.701

21. Ulusoy G., Kılıç K., Günday G., Alpkan L. A determinants of innovativeness model for manufacturing firms. International Journal of Innovation and Regional Development. 2015;6(2):125158. DOI: $10.1504 /$ IJIRD.2015.069662

22. Boschma R.A., Ter Wal A.L. Knowledge networks and innovative performance in an industrial district: The case of a footwear district in the South of Italy. Industry and Innovation. 2007;14(2):177-199.

23. Schumpeter J.A. Change and the entrepreneur. In: Clemence R.V., ed. Essays of J.A. Schumpeter. Cambridge, MA: Addison-Wesley; 1934.

24. Spieth P., Schneider S. Business model innovativeness: designing a formative measure for business model innovation. Journal of Business Economics. 2016;86(6):671-696. DOI: 10.1007/ s11573-015-0794-0

25. Geldes C., Felzensztein C., Palacios-Fenech J. Technological and non-technological innovations, performance and propensity to innovate across industries: The case of an emerging economy. Industrial Marketing Management. 2017;61:55-66. DOI: 10.1016/j.indmarman.2016.10.010

26. Lee J.S., Park J.H., Bae Z.T. The effects of licensing-in on innovative performance in different technological regimes. Research Policy. 2017;46(2):485-496. DOI: 10.1016/j.respol.2016.12.002

27. Alcaniz L., Gomez-Bezares F., Roslender R. Theoretical perspectives on intellectual capital: A backward look and a proposal for going forward. Accounting Forum. 2011;35(2):104-117. DOI: 10.1016/j.accfor.2011.03.004
28. McGuirk H., Lenihan H., Hart M. Measuring the impact of innovative human capital on small firms' propensity to innovate. Research Policy. 2015;44(4):965-976. DOI: 10.1016/j. respol.2014.11.008

29. Calantone R.J., Cavusgil S.T., Zhao Y. Learning orientation, firm innovation capability, and firm performance. Industrial Marketing Management. 2002;31(6):515-524. DOI: 10.1016/S00198501(01)00203-6

30. Caloghirou Y., Kastelli I., Tsakanikas A. Internal capabilities and external knowledge sources: Complements or substitutes for innovative performance? Technovation. 2004;24(1):29-39. DOI: 10.1016/S0166-4972(02)00051-2

31. Martynova M., Renneboog L. Spillover of corporate governance standards in cross-border mergers and acquisitions. Journal of Corporate Finance. 2008;14(3):200-223. DOI: 10.1016/j. jcorpfin.2008.03.004

32. Chatterjee S. Types of synergy and economic value: The impact of acquisitions on merging and rival firms. Strategic Management Journal. 1986;7(2):119139. DOI: $10.1002 / \mathrm{smj} .4250070203$

33. Chiara Di Guardo M., Valentini G. Explaining the effect of M\&A on technological performance. In: Cooper C.L., Finkelstein S., eds. Advances in Mergers and Acquisitions. Vol. 6. Jordan Hill, Amsterdam, San Diego: JAI Press; 2007:107-125.

34. Datta D.K., Puia G. Cross-border acquisitions: An examination of the influence of relatedness and cultural fit on shareholder value creation in US acquiring firms. MIR: Management International Review. 1995;35(4):337-359.

35. Bauer F., Matzler K. Antecedents of M\&A success: The role of strategic complementarity, cultural fit, and degree and speed of integration. Strategic Management Journal. 2014;35(2):269-291. DOI: 10.1002/smj.2091

36. Capron L., Dussauge P., Mitchell W. Resource redeployment following horizontal acquisitions in Europe and North America, 1988-1992. Strategic Management Journal. 1998;19(7):631-661. DOI: 10.1002/(SICI)1097-0266(199807)19:7<631::AIDSMJ963>3.0.CO;2-9

37. Walter G.A., Barney J.B. Research notes and communications management objectives in mergers and acquisitions. Strategic Management Journal. 1990;11(1):79-86. DOI: 10.1002/smj.4250110107

38. Miozzo M., DiVito L., Desyllas P. When do acquirers invest in the R\&D assets of acquired science-based firms in cross-border acquisitions? The role of technology and capabilities similarity and complementarity. Long Range Planning. 2016;49(2):221-240. DOI: 10.1016/j.lrp.2015.07.002 
39. Seth A. Value creation in acquisitions: A reexamination of performance issues Strategic Management Journal. 1990;11(2):99-115. DOI: 10.1002/smj.4250110203

40. DiMasi J.A., Hansen R.W., Grabowski H.G. The price of innovation: new estimates of drug development costs. Journal of Health Economics. 2003;22(2):151185. DOI: 10.1016/S0167-6296(02)00126-1

41. Chatterjee S., Lubatkin M. Corporate mergers, stockholder diversification, and changes in systematic risk. Strategic Management Journal. 1990;11(4):255268. DOI: $10.1002 / \mathrm{smj} .4250110402$

42. Leland H.E. Financial synergies and the optimal scope of the firm: Implications for mergers, spinoffs, and structured finance. The Journal of Finance. 2007;62(2):765-807. DOI: 10.1111/j.15406261.2007.01223.x

43. De Man A.P., Duysters G. Collaboration and innovation: a review of the effects of mergers, acquisitions and alliances on innovation. Technovation. 2005;25(12):1377-1387. DOI: 10.1016/j.technovation.2004.07.021

44. Gubbi S.R., Aulakh P.S., Ray S., Sarkar M.B., Chittoor R. Do international acquisitions by emergingeconomy firms create shareholder value? The case of Indian firms. Journal of International Business Studies. 2010;41(3):397-418. DOI: 10.1057/jibs.2009.47

45. Ahuja G., Katila R. Technological acquisitions and the innovation performance of acquiring firms: A longitudinal study. Strategic Management Journal. 2001;22(3):197-220. DOI: 10.1002/smj.157

46. Bena J., Li K. Corporate innovations and mergers and acquisitions. The Journal of Finance. 2014;69(5):19231960. DOI: $10.1111 /$ jofi.12059

47. Roll R. The hubris hypothesis of corporate takeovers. Journal of Business. 1986;59(2):197-216.

48. Shleifer A., Vishny R.W. Management entrenchment: The case of manager-specific investments. Journal of Financial Economics. 1989;25(1):123-139. DOI: 10.1016/0304-405X(89)90099-8

49. Kohers N., Kohers T. The value creation potential of high-tech mergers. Financial Analysts Journal. 2000;56(3):40-51. DOI: DOI: 10.2469/faj.v56.n3.2359

50. Hagedoorn J., Duysters G. External sources of innovative capabilities: The preferences for strategic alliances or mergers and acquisitions. Journal of Management Studies. 2002;39(2):167-188. DOI: 10.1111/1467-6486.00287

51. Cloodt M., Hagedoorn J., Van Kranenburg H. Mergers and acquisitions: Their effect on the innovative performance of companies in high-tech industries. Research Policy. 2006;35(5):642-654. DOI: 10.1016/j.respol.2006.02.007
52. Valentini G. Measuring the effect of M\&A on patenting quantity and quality. Strategic Management Journal. 2012;33(3):336-346. DOI: 10.1002/smj.946

53. Colombo M.G., Rabbiosi L. Technological similarity, post-acquisition $\mathrm{R} \& \mathrm{D}$ reorganization, and innovation performance in horizontal acquisitions. Research Policy. 2014;43(6):1039-1054. DOI: 10.1016/j. respol.2014.01.013

54. Cefis E., Marsili O. Crossing the innovation threshold through mergers and acquisitions. Research Policy. 2015;44(3):698-710. DOI: 10.1016/j.respol.2014.10.010

55. Stiebale J., Reize F. The impact of FDI through mergers and acquisitions on innovation in target firms. International Journal of Industrial Organization. 2011;29(2):155-167. DOI: 10.1016/j. ijindorg.2010.06.003

56. Patel P.C., King D. Managing cultural distance: effects of technological coherence and diversity in crossborder acquisitions. Working Paper. 2011.

57. Pradhan J.P. Strategic asset-seeking activities of emerging multinationals: Perspectives on foreign acquisitions by Indian pharmaceutical MNEs. Organizations and Markets in Emerging Economies. 2010;1(2):9-31.

58. Yu Y., Dong X.Y., Shen K.N., Khalifa M., Hao J.X. Strategies, technologies, and organizational learning for developing organizational innovativeness in emerging economies. Journal of Business Research. 2013;66(12):2507-2514. DOI: 10.1016/j. jbusres.2013.05.042

59. De Beule F., Sels A. Do innovative emerging market cross-border acquirers create more shareholder value? Evidence from India. International Business Review. 2016;25(2):604-617. DOI: 10.1016/j. ibusrev.2015.09.008

60. Anderson J., Sutherland D., Severe S. An event study of home and host country patent generation in Chinese MNEs undertaking strategic asset acquisitions in developed markets. International Business Review. 2015;24(5):758-771. DOI: 10.1016/j.ibusrev.2015.01.007

61. Awate S., Larsen M.M., Mudambi R. EMNE catch-up strategies in the wind turbine industry: Is there a trade-off between output and innovation capabilities? Global Strategy Journal. 2012;2(3):205-223. DOI: 10.1111/j.2042-5805.2012.01034.x

62. Deng P. Why do Chinese firms tend to acquire strategic assets in international expansion? Journal of World Business. 2009;44(1):74-84. DOI: 10.1016/j. jwb.2008.03.014

63. Cheng C., Yang M. Enhancing performance of cross-border mergers and acquisitions in developed markets: The role of business ties and technological innovation capability. Journal of Business Research. 2017;81:107-117. DOI: 10.1016/j.jbusres.2017.08.019 


\title{
Mergers and Acquisitions of
}

\section{Innovative Companies in Developed and Emerging Capital Markets: What Sets Them Apart?}

\author{
Irina Skvortsova \\ Lecturer, Faculty of Economic Sciences, School of Finance \\ National Research University Higher School of Economics \\ 20 Myasnitskaya St, Moscow, Russia \\ E-mail: irina.v.skvortsova@gmail.com
}

\author{
Andrey Krasovitskiy \\ Research Intern, Scientific Research Laboratory of Corporate Finance \\ National Research University Higher School of Economics \\ 20 Myasnitskaya St, Moscow, Russia \\ E-mail: andreykras99@gmail.com
}

\begin{abstract}
Currently, innovative companies are one of the key drivers of the global market for mergers and acquisitions. This makes it important to identify their specific features, as well as to study the effectiveness of such deals. However, in modern academic literature there is no definitive answer to the question of which company can be considered innovative, and how to determine its level of innovation. Also, there is no clear answer to the question of what an innovative M\&A deal is, and what distinguishing features it possesses. Extremely little attention is paid to identifying cross-border innovative mergers and acquisitions transactions and the determination of their distinct features. This paper provides a brief overview of the available literature on these topics. It describes how to define an innovative company and the main approaches to assess the effectiveness of innovative mergers and acquisitions deals. It also discusses international innovative mergers and acquisitions and their features. Based on the results of the literature review, it was concluded that in most of the works, innovation is determined through the absolute value of various indicators listed in this paper, or with the help of special questionnaires sent to the company's management. Innovative companies can also be defined based on the industries in which companies are involved. Companies' innovative performance after mergers and acquisitions is measured by a change in the absolute quantity of company patents, or in the quantity of citations of existing company patents. It was not possible to establish a clear answer about the impact of mergers and acquisitions on the innovative performance of the company, since the research indicates contradictory results. The main motive of crossborder innovation M\&A deals is the acquisition of new technologies to gain competitive advantages. However, there is no unequivocal effect on innovation performance as a result of these type of transactions.
\end{abstract}

Keywords: mergers and acquisitions, innovative companies, innovations, innovative performance, company performance, developed markets, emerging markets

JEL: G34, L10, O30, O31 


\section{References}

1. Quinn J.B. Technological innovation, entrepreneurship, and strategy. IEEE Engineering Management Review. 1983;20(3):14-25. DOI: 10.1109/EMR.1983.4306000

2. Damanpour F. Organizational innovation: A metaanalysis of effects of determinants and moderators. Academy of Management Journal. 1991;34(3):555590. DOI: $10.5465 / 256406$

3. Damanpour F. Organizational complexity and innovation: Developing and testing multiple contingency models. Management Science. 1996;42(5):693-716. DOI: 10.1287/mnsc.42.5.693

4. Tidd J., Bessant J., Pavitt K. et al. Management dell'innovazione: L'integrazione del cambiamento tecnologico, organizzativo e dei mercati. Milano: Guerini e associate; 1999. 453 p.

5. Valls J.P., Escorsa P. Tecnología e innovación en la empresa: Dirección y gestión. Barcelona: Universidad Politecnica de Cataluny; 2000. 341 p.

6. Camisón-Zornoza C., Lapiedra-Alcamí R., Segarra-Ciprés M., Boronat-Navarro M. A metaanalysis of innovation and organizational size. Organization Studies. 2004;25(3):331-361. DOI: $10.1177 / 0170840604040039$

7. Subramanian A., Nilakanta S. Organizational innovativeness: Exploring the relationship between organizational determinants of innovation, types of innovations, and measures of organizational performance. Omega. 1996;24(6):631-647. DOI: 10.1016/S0305-0483(96)00031-X

8. Azar G., Ciabuschi F. Organizational innovation, technological innovation, and export performance: The effects of innovation radicalness and extensiveness. International Business Review. 2017;26(2):324-336. DOI: 10.1016/j. ibusrev.2016.09.002

9. Leicht T., Chtourou A., Youssef K.B. Consumer innovativeness and intentioned autonomous car adoption. The Journal of High Technology Management Research. 2018;29(1):1-11. DOI: 10.1016/j.hitech.2018.04.001

10. Hagedoorn J., Cloodt M. Measuring innovative performance: is there an advantage in using multiple indicators? Research Policy. 2003;32(8):1365-1379. DOI: 10.1016/S0048-7333(02)00137-3

11. OECD, 2005. Oslo Manual, OECD, Paris.

12. Hall L.A., Bagchi-Sen S. A study of R\&D, innovation, and business performance in the Canadian biotechnology industry. Technovation. 2002;22(4):231-244. DOI: 10.1016/S01664972(01)00016-5
13. Artz K.W., Norman P.M., Hatfield D.E., Cardinal L.B. A longitudinal study of the impact of R\&D, patents, and product innovation on firm performance. The Journal of Product Innovation Management. 2010;27(5):725-740. DOI: 10.1111/j.15405885.2010.00747.x

14. Dutta S., Weiss A.M. The relationship between a firm's level of technological innovativeness and its pattern of partnership agreements. Management Science. 1997;43(3):257-402. DOI: 10.1287/ mnsc. 43.3 .343

15. Hult G.T.M., Hurley R.F., Knight G.A. Innovativeness: Its antecedents and impact on business performance. Industrial Marketing Management. 2004;33(5):429438. DOI: 10.1016/j.indmarman.2003.08.015

16. Sydler R., Haefliger S., Pruksa R. Measuring intellectual capital with financial figures: Can we predict firm profitability? European Management Journal. 2014;32(2):244-259. DOI: 10.1016/j. emj.2013.01.008

17. Fung M.K. Is innovativeness a link between pay and performance? Financial Management. 2009;38(2):411-429. DOI: 10.1111/j.1755053X.2009.01041.X

18. Leitner S.M., Stehrer R. The role of financial constraints for different innovation strategies: Evidence for CESEE and FSU countries. Wiener Institut für Internationale Wirtschaftsvergleiche. WIIW Working Paper. 2016;(125). URL: https://wiiw. ac.at/the-role-of-financial-constraints-for-differentinnovation-strategies-evidence-for-cesee-and-fsucountries-dlp-3875.pdf

19. Shi X., Wu Y. The effect of internal and external factors on innovative behaviour of Chinese manufacturing firms. China Economic Review. 2017;46(S):S50-S64. DOI: 10.1016/j. chieco.2016.08.010

20. Aghion P., Bloom N., Blundell R., Griffith R., Howitt P. Competition and innovation: An inverted-U relationship. The Quarterly Journal of Economics. 2005;120(2):701-728. DOI: 10.1093/qje/120.2.701

21. Ulusoy G., Kılıç K., Günday G., Alpkan L. A determinants of innovativeness model for manufacturing firms. International Journal of Innovation and Regional Development. 2015;6(2):125158. DOI: 10.1504/IJIRD.2015.069662

22. Boschma R.A., Ter Wal A.L. Knowledge networks and innovative performance in an industrial district: The case of a footwear district in the South of Italy. Industry and Innovation. 2007;14(2):177-199.

23. Schumpeter J.A. Change and the entrepreneur. In: Clemence R.V., ed. Essays of J.A. Schumpeter. Cambridge, MA: Addison-Wesley; 1934. 
24. Spieth P., Schneider S. Business model innovativeness: designing a formative measure for business model innovation. Journal of Business Economics. 2016;86(6):671-696. DOI: 10.1007/ s11573-015-0794-0

25. Geldes C., Felzensztein C., Palacios-Fenech J. Technological and non-technological innovations, performance and propensity to innovate across industries: The case of an emerging economy. Industrial Marketing Management. 2017;61:55-66. DOI: 10.1016/j.indmarman.2016.10.010

26. Lee J.S., Park J.H., Bae Z.T. The effects of licensing-in on innovative performance in different technological regimes. Research Policy. 2017;46(2):485-496. DOI: 10.1016/j.respol.2016.12.002

27. Alcaniz L., Gomez-Bezares F., Roslender R. Theoretical perspectives on intellectual capital: A backward look and a proposal for going forward. Accounting Forum. 2011;35(2):104-117. DOI: 10.1016/j.accfor.2011.03.004

28. McGuirk H., Lenihan H., Hart M. Measuring the impact of innovative human capital on small firms' propensity to innovate. Research Policy. 2015;44(4):965-976. DOI: 10.1016/j. respol.2014.11.008

29. Calantone R.J., Cavusgil S.T., Zhao Y. Learning orientation, firm innovation capability, and firm performance. Industrial Marketing Management. 2002;31(6):515-524. DOI: 10.1016/S00198501(01)00203-6

30. Caloghirou Y., Kastelli I., Tsakanikas A. Internal capabilities and external knowledge sources: Complements or substitutes for innovative performance? Technovation. 2004;24(1):29-39. DOI: 10.1016/S0166-4972(02)00051-2

31. Martynova M., Renneboog L. Spillover of corporate governance standards in cross-border mergers and acquisitions. Journal of Corporate Finance. 2008;14(3):200-223. DOI: 10.1016/j. jcorpfin.2008.03.004

32. Chatterjee S. Types of synergy and economic value: The impact of acquisitions on merging and rival firms. Strategic Management Journal. 1986;7(2):119139. DOI: $10.1002 / \mathrm{smj} .4250070203$

33. Chiara Di Guardo M., Valentini G. Explaining the effect of M\&A on technological performance. In: Cooper C.L., Finkelstein S., eds. Advances in Mergers and Acquisitions. Vol. 6. Jordan Hill, Amsterdam, San Diego: JAI Press; 2007:107-125.

34. Datta D.K., Puia G. Cross-border acquisitions: An examination of the influence of relatedness and cultural fit on shareholder value creation in US acquiring firms. MIR: Management International Review. 1995;35(4):337-359.
35. Bauer F., Matzler K. Antecedents of M\&A success: The role of strategic complementarity, cultural fit, and degree and speed of integration. Strategic Management Journal. 2014;35(2):269-291. DOI: 10.1002/smj.2091

36. Capron L., Dussauge P., Mitchell W. Resource redeployment following horizontal acquisitions in Europe and North America, 1988-1992. Strategic Management Journal. 1998;19(7):631-661. DOI: 10.1002/(SICI)1097-0266(199807)19:7<631::AIDSMJ963>3.0.CO;2-9

37. Walter G.A., Barney J.B. Research notes and communications management objectives in mergers and acquisitions. Strategic Management Journal. 1990;11(1):79-86. DOI: 10.1002/smj.4250110107

38. Miozzo M., DiVito L., Desyllas P. When do acquirers invest in the R\&D assets of acquired science-based firms in cross-border acquisitions? The role of technology and capabilities similarity and complementarity. Long Range Planning. 2016;49(2):221-240. DOI: 10.1016/j.lrp.2015.07.002

39. Seth A. Value creation in acquisitions: A reexamination of performance issues Strategic Management Journal. 1990;11(2):99-115. DOI: $10.1002 /$ smj.4250110203

40. DiMasi J.A., Hansen R.W., Grabowski H.G. The price of innovation: new estimates of drug development costs. Journal of Health Economics. 2003;22(2):151185. DOI: 10.1016/S0167-6296(02)00126-1

41. Chatterjee S., Lubatkin M. Corporate mergers, stockholder diversification, and changes in systematic risk. Strategic Management Journal. 1990;11(4):255268. DOI: $10.1002 / \mathrm{smj} .4250110402$

42. Leland H.E. Financial synergies and the optimal scope of the firm: Implications for mergers, spinoffs, and structured finance. The Journal of Finance. 2007;62(2):765-807. DOI: 10.1111/j.15406261.2007.01223.x

43. De Man A.P., Duysters G. Collaboration and innovation: a review of the effects of mergers, acquisitions and alliances on innovation. Technovation. 2005;25(12):1377-1387. DOI: 10.1016/j.technovation.2004.07.021

44. Gubbi S.R., Aulakh P.S., Ray S., Sarkar M.B., Chittoor R. Do international acquisitions by emergingeconomy firms create shareholder value? The case of Indian firms. Journal of International Business Studies. 2010;41(3):397-418. DOI: 10.1057/jibs.2009.47

45. Ahuja G., Katila R. Technological acquisitions and the innovation performance of acquiring firms: A longitudinal study. Strategic Management Journal. 2001;22(3):197-220.

DOI: $10.1002 /$ smj.157 
46. Bena J., Li K. Corporate innovations and mergers and acquisitions. The Journal of Finance. 2014;69(5):19231960. DOI: $10.1111 /$ jofi.12059

47. Roll R. The hubris hypothesis of corporate takeovers. Journal of Business. 1986;59(2):197-216.

48. Shleifer A., Vishny R.W. Management entrenchment: The case of manager-specific investments. Journal of Financial Economics. 1989;25(1):123-139. DOI: 10.1016/0304-405X(89)90099-8

49. Kohers N., Kohers T. The value creation potential of high-tech mergers. Financial Analysts Journal. 2000;56(3):40-51. DOI: DOI: 10.2469/faj.v56.n3.2359

50. Hagedoorn J., Duysters G. External sources of innovative capabilities: The preferences for strategic alliances or mergers and acquisitions. Journal of Management Studies. 2002;39(2):167-188. DOI: $10.1111 / 1467-6486.00287$

51. Cloodt M., Hagedoorn J., Van Kranenburg H. Mergers and acquisitions: Their effect on the innovative performance of companies in high-tech industries. Research Policy. 2006;35(5):642-654. DOI: 10.1016/j.respol.2006.02.007

52. Valentini G. Measuring the effect of M\&A on patenting quantity and quality. Strategic Management Journal. 2012;33(3):336-346. DOI: 10.1002/smj.946

53. Colombo M.G., Rabbiosi L. Technological similarity, post-acquisition $R \& D$ reorganization, and innovation performance in horizontal acquisitions. Research Policy. 2014;43(6):1039-1054. DOI: 10.1016/j. respol.2014.01.013

54. Cefis E., Marsili O. Crossing the innovation threshold through mergers and acquisitions. Research Policy. 2015;44(3):698-710. DOI: 10.1016/j. respol.2014.10.010

55. Stiebale J., Reize F. The impact of FDI through mergers and acquisitions on innovation in target firms. International Journal of Industrial Organization. 2011;29(2):155-167. DOI: 10.1016/j. ijindorg.2010.06.003
56. Patel P.C., King D. Managing cultural distance: effects of technological coherence and diversity in crossborder acquisitions. Working Paper. 2011.

57. Pradhan J.P. Strategic asset-seeking activities of emerging multinationals: Perspectives on foreign acquisitions by Indian pharmaceutical MNEs. Organizations and Markets in Emerging Economies. 2010;1(2):9-31.

58. Yu Y., Dong X.Y., Shen K.N., Khalifa M., Hao J.X. Strategies, technologies, and organizational learning for developing organizational innovativeness in emerging economies. Journal of Business Research. 2013;66(12):2507-2514. DOI: 10.1016/j. jbusres.2013.05.042

59. De Beule F., Sels A. Do innovative emerging market cross-border acquirers create more shareholder value? Evidence from India. International Business Review. 2016;25(2):604-617. DOI: 10.1016/j. ibusrev.2015.09.008

60. Anderson J., Sutherland D., Severe S. An event study of home and host country patent generation in Chinese MNEs undertaking strategic asset acquisitions in developed markets. International Business Review. 2015;24(5):758-771. DOI: 10.1016/j. ibusrev.2015.01.007

61. Awate S., Larsen M.M., Mudambi R. EMNE catch-up strategies in the wind turbine industry: Is there a trade-off between output and innovation capabilities? Global Strategy Journal. 2012;2(3):205-223. DOI: 10.1111/j.2042-5805.2012.01034.x

62. Deng P. Why do Chinese firms tend to acquire strategic assets in international expansion? Journal of World Business. 2009;44(1):74-84. DOI: 10.1016/j. jwb.2008.03.014

63. Cheng C., Yang M. Enhancing performance of cross-border mergers and acquisitions in developed markets: The role of business ties and technological innovation capability. Journal of Business Research. 2017;81:107-117. DOI: 10.1016/j.jbusres.2017.08.019 